British Journal of Psychiatry (1988), 153, 700-715

\title{
Correspondence
}

Editor: Ian Pullen

Contents: Personality disorder/Psychiatric morbidity in the Territorial Army/Screening for HIV/ Emotional disturbances in endocrine patients/Geographical error/Melatonin secretion in depression/ Self-esteem: a psychiatric view/Depersonalisation and self-perception/Lithium-induced carpal tunnel syndrome/The truth about ECT/Is castration too "barbarous" for rapists?/Females and caring/Delusional depression in nineteenth century Scotland/ Koro secondary to a tumour of the corpus callosum/ A capital mistake?/Sherlock Holmes - would he be a good psychoanalyst?/50 years of cocaine addiction/ Shoplifting as a symptom of stress in families of mentally handicapped persons: a case report/Auditory hallucinations due to ear disease/Anorexia nervosa and infantile autism/Capgras syndrome and organic disease.

\section{Personality Disorder}

SIR: I would like to congratulate Lewis \& Appleby (Journal, July 1988, 153,44-49) on a neat and interesting paper. It tackles a fundamental problem in modern psychiatry. In clinical practice it is disturbance of personality which is one of our biggest challenges. As ICD-9 acknowledges, even schizophrenia is "a fundamental disturbance of personality", and it is the management of the personality disturbance which is one of the most difficult aspects of treating that or any other disorder.

I entirely agree with Drs Lewis \& Appleby that part of the problem with our modern understanding of the concept of personality disorder is that it is regarded as something other than an illness and therefore inexcusable. An aspect of this process may be the use of the curious phrase "no formal mental illness" in medical reports on highly abnormal individuals. What, I ask myself, is an informal mental illness? It could be that the word formal here is used to acknowledge the disorder while simultaneously barring that disorder from psychiatric help because it is inexcusable. It could also be that this device is used by the psychiatrist to escape the agonies and responsibilities of assisting difficult patients with protracted disorders that do not clear up.
Drs Lewis \& Appleby do not speculate on the reasons for 'mental illness' to be associated with favourable psychiatric attitudes and 'personality disorder' to be associated with unfavourable ones. I would like to suggest that one important factor is that we currently believe that 'illnesses', such as depression, schizophrenia, and mania, have 'treatments' in the form of medication, but that personality disorders are 'untreatable'. Another factor is that patients with long-standing traits such as anhedonia, dependency, and aggressiveness are diffcult to like. It is for this latter reason that I disagree slightly with Lewis \& Appleby's final conclusion, proposing the abandonment of the term personality disorder. Changing names for objects that are disliked is a common ploy, but the stigma soon attaches itself to the new name: cf. water closet, lavatory, toilet, or subnormality, handicap, impairment. A more taxing approach, but one which may have a bit more success in the long run, is to change psychiatric attitudes through education.

Another device, which combines name changing with an improved clinical technique, might be to provide each patient with a personal function analysis in the same way that we currently provide each patient with a mental state analysis. This would give a list of functions with varying degrees of mutability and thus draw attention more clearly to treatment potentials. I have argued for this approach in greater length in a paper about to be published (Gunn, 1988).

J. GUNN

Institute of Psychiatry

De Crespigny Park

Denmark Hill

London SE5 8AF

\section{Reforence}

GuNN, J. C. (1988) Personality disorder: a clinical suggestion. In Personality Disorders: Diagnosis, Management and Course (ed. P. Tyrer). London: Butterworths (in press).

SIR: Our contemporary, existentialist, society is reluctant to pass moral judgements, and perhaps this underlies the assertion of Drs Lewis \& Appleby that the diagnosis of personality disorder should 
be discarded, because it constitutes 'an enduring pejorative judgement'. This point of view would be laudable if they had shown that the diagnosis could not be reliably made, or that it held no validity, but this they have not done.

Drs Lewis \& Appleby have shown that the diagnosis of personality disorder conveys to psychiatrists a negative moral connotation, presumably by virtue of its attribution of individual responsibility for socially unacceptable behaviour. As with hysteria (Miller, 1988), this attribution of responsibility may well be difficult to prove. However, the issue is an important one, since patients who are responsible for such behaviour may not be helped by being treated as 'sick'. The moral judgement which follows this line of reasoning, assuming it is valid, is no more or less deserved than that passed on others who display similar behaviour without receiving the diagnostic label of personality disorder. Debt (or lying or theft or a range of other types of behaviour) should only be deemed exempt from moral judgement where attribution of personal responsibility is waived, as for example in severe depression or dementia. If patients with personality disorder are responsible for their behaviour, then moral judgements are not misplaced. If they are not responsible, then such judgements should not be made. Drs Lewis and Appleby, however, have not addressed this issue.

Surely a more worrying conclusion to be drawn from this study is that the consequences of diagnosing personality disorder are far-reaching. Once the label is given it is not easily removed, and attempts to diagnose or treat psychiatric illnesses such as depression are reduced or even abandoned. Patients with a personality disorder (or patients without one who are so misdiagnosed) thus appear to have been made responsible also for behaviour which would in others have been excused! Worse still, they have effectively been denied the benefit of potentially efficacious treatments.

If the diagnosis of personality disorder can be shown to warrant exemption from the usual attribution of responsibility for socially unacceptable behaviour, then psychiatrists and others must change their judgemental attitudes. While the validity of this diagnosis and the reliability of its application remain in doubt, it should be used only with good reason and great caution. Only when it has been shown that the concept of personality disorder holds no validity should it be discarded.

C. C. H. CoOK

University College \& Middlesex School of Medicine Middlesex Hospital

London WIN 8AA
Reference

MiLler, E. (1988) Defining hysterical symptoms. Psychological Medicine, 18, 275-277.

SIR: Drs Lewis \& Appleby argue that because psychiatrists are unable to resist expressing their hostility towards the personality disordered (PD) patient by rejecting him or her the concept is invalid. It is the poor workman who blames his tools. Thus it is a false argument to abandon the concept of PD because psychiatrists feel uneasy in the face of the reality the concept represents. Rather, psychiatrists should examine their bad practice and understand it.

Psychiatrists, like other professionals, have poor tolerance of situations in which their accustomed sense of power, authority, and efficacy is eroded. With PD patients it is often the doctor who suffers and the patient who acts (out). The PD patient challenges the psychiatrist because, before therapy can even be attempted, extensive negiotiations have to be entered into (often of a contractual nature) with the patient as equal negotiator. This is far different from other clinical situations in which the patient is, by and large, the sufferer and forms the passive side to a markedly unequal power relationship. Moreover, the time scale in which therapy may need to be attempted is immensely long - as likely 10 years as 10 months - which again is not guaranteed to gratify the therapist's self-esteem or desire for readily observable results.

It is fundamentally misconceived to attempt to subsume PD under the concept of mental illness with "a classification based on symptoms". Historically PD has always been recognised as a separate entity, although the starting point of definition has been a comparison with mental illness, e.g. Pinel's manie sans délire. There is a suggestion that cultures quite different from our own recognise PD separately from mental illness (Murphy, 1976). The Eskimo term kunlangeta, meaning "his mind knows what to do but he does not do it", and the Yoruba term arankan. meaning a person who goes his own way regardless of others, both describe deviations from social and personal norms which increase the individual's tendency to be vulnerable to illness or other misfortune. Interestingly enough these disorders are not believed to respond to the conventional healer's techniques.

I would also disagree that a psychiatric classification loses credibility if it "contains value judgements or moral statements", since the latter are inseparable from and implicit in the human as opposed to the physical sciences. The psychiatrist's feeling of dislike and hostility is an important piece 